\title{
A GENERAL METHOD TO OBTAIN THE SPECTRUM AND LOCAL SPECTRA OF A GRAPH FROM ITS REGULAR PARTITIONS*
}

\author{
CRISTINA DALFÓ ${ }^{\dagger}$ AND MIQUEL ÀNGEL FIOL ${ }^{\ddagger}$
}

\begin{abstract}
It is well known that, in general, part of the spectrum of a graph can be obtained from the adjacency matrix of its quotient graph given by a regular partition. In this paper, a method that gives all the spectrum, and also the local spectra, of a graph from the quotient matrices of some of its regular partitions, is proposed. Moreover, from such partitions, the $C$-local multiplicities of any class of vertices $C$ is also determined, and some applications of these parameters in the characterization of completely regular codes and their inner distributions are described. As examples, it is shown how to find the eigenvalues and (local) multiplicities of walk-regular, distance-regular, and distance-biregular graphs.
\end{abstract}

Key words. Adjacency matrix, Spectrum, Eigenvalues, Local multiplicities, Walk-regular graph, $C$-local spectrum, Completely regular code.

AMS subject classifications. 05C50, 05E30.

1. Introduction. It is well known that the (adjacency) spectrum of a graph $\Gamma$ does not determine, in general, its whole structure. However, many combinatorial properties of $\Gamma$ can be derived from its spectrum. See, for example, the comprehensive textbooks of Biggs [1], Cvetković, Doob, and Sachs [6], Cvetković, Rowlinson, and Simić [7], and Brouwer and Haemers [3]. In particular, a persisting problem is to show whether or not a given graph is completely determined by its spectrum (see van Dam and Haemers [11]), which has led to the following conjecture.

CONJECTURE 1.1. ([11]) Almost every graph is determined by its spectrum.

The table below, showing the ratio of graphs on $n \leq 12$ vertices not determined by their spectrum ('n-DS') to all graphs of order $n$, may be taken as a support of this conjecture.

\begin{tabular}{|c||cccccccccccc|}
\hline$n$ & 1 & 2 & 3 & 4 & 5 & 6 & 7 & 8 & 9 & 10 & 11 & 12 \\
\hline n-DS & 0 & 0 & 0 & 0 & 0.059 & 0.064 & 0.105 & 0.139 & 0.186 & 0.213 & 0.211 & 0.188 \\
\hline
\end{tabular}

Thus, there is a big area of research about trying to find, in an efficient way, the spectrum (or at least a part of it) for infinite families of graphs. In particular, some methods to do so are known for graphs with high symmetry and/or regularity. These include, for instance, the distance-regular graphs, Cayley graphs, lifted graphs, etc. In contrast, much less is known about finding the so-called 'local spectrum' (with local eigenvalues and multiplicities associated) of a vertex, and the 'crossed spectrum' (with crossed multiplici-

\footnotetext{
* Received by the editors on February 20, 2020. Accepted for publication on May 27, 2020. Handling Editor: Sebastian M. Cioaba. Corresponding Author: Cristina Dalfó.

${ }^{\dagger}$ Departament de Matemàtica, Universitat de Lleida, Igualada (Barcelona), Catalonia (cristina.dalfo@udl.cat). Partially supported by AGAUR from the Catalan Government under project 2017SGR1087 and by MICINN from the Spanish Government under projects PGC2018-095471-B-I00 and MTM2017-83271-R. Also received funding from the European Union's Horizon 2020 research and innovation programme under the Marie Skłodowska-Curie grant agreement no. 734922.

${ }^{\ddagger}$ Departament de Matemàtiques, Barcelona Graduate School of Mathematics, Universitat Politècnica de Catalunya, Barcelona, Catalonia, (miguel.angel.fiol@upc.edu). Partially supported by AGAUR from the Catalan Government under project 2017SGR1087 and by MICINN from the Spanish Government under project PGC2018-095471-B-I00.
} 
447 A General Method to Obtain the Spectrum and Local Spectra of a Graph from its Regular Partitions

ties eigenvalues associated with pairs of vertices). The theory of graph (global) spectra has applications in computer science (for instance, in data structure and quantum computing), quantum chemistry, electrical engineering, game theory, etc. Moreover, the local (adjacency or Laplacian) spectra have been a key concept to derive reliable theoretical results, as the so-called 'spectral excess theorem' (a quasi-spectral characterization of distance-regular graphs due to Fiol and Garriga [17]), and its sequels, see, for instance, Kurihara [24]. Besides, the knowledge of local spectra of a complex network provides essential insight into its topology and dynamical behavior, see Moradiamani, Fiol, Jalili, Stone, Chen, and Yu [27].

Given a general graph $\Gamma$ and a regular partition of its vertex set, we can obtain a smaller quotient graph with its (adjacency) quotient matrix. The spectrum of this matrix gives us a part of the spectrum of the original graph. In this paper, we propose a method that provides not only a part but all the spectrum, and also the local spectra, of $\Gamma$, by using the quotient matrices of some of its regular partitions.

This paper is organized as follows. In this section, we recall the concepts of the idempotent matrix, the local multiplicity of an eigenvalue associated with a vertex, the local spectrum of a graph, and the local crossed multiplicity. In Section 2, we remind the definition of a regular partition and some known lemmas. Moreover, also in this section, we give some new lemmas and the main result. Some of the previous results are generalized in Section 3, where we deal with regular partitions and local spectra of vertex subsets. We also describe some applications in the characterization of completely regular codes. Finally, in Section 4, we apply our method to find the eigenvalues, (local) multiplicities, and the complete spectrum of three families of graphs, which are walk-regular, distance-regular, and distance-biregular graphs.

1.1. Some notions of graphs and their spectra. First, let us recall some basic concepts and define our generic notation for graphs.

Throughout this paper, $\Gamma=(V, E)$ denotes a simple and connected graph with order $n=|V|$, size $m=|E|$, and adjacency matrix $\boldsymbol{A}$. The distance between two vertices $u$ and $v$ is denoted by $\operatorname{dist}(u, v)$, so that the eccentricity of a vertex $u$ is $\operatorname{ecc}(u)=\max _{v \in V} \operatorname{dist}(u, v)$, and the diameter of the graph is $D=\max _{u \in V} \operatorname{ecc}(u)$. The set of vertices at distance $i$, from a given vertex $u \in V$ is denoted by $\Gamma_{i}(u)$, for $i=0,1, \ldots, D$, and we write $\Gamma(u)=\Gamma_{1}(u)$ for short. The degree of a vertex $u$ is denoted by $\delta(u)=\left|\Gamma_{1}(u)\right|$. The distance- $i$ graph $\Gamma_{i}$ is the graph with vertex set $V$, and where two vertices $u$ and $v$ are adjacent if and only if $\operatorname{dist}(u, v)=i$ in $\Gamma$. Its adjacency matrix $\boldsymbol{A}_{i}$ is usually referred to as the distance- $i$ matrix of $\Gamma$ and, if $\Gamma$ is distance-regular, we have $\boldsymbol{A}_{i}=p_{i}(\boldsymbol{A})$, where $p_{i}, i=0, \ldots, d$, are the so-called distance polynomials of $\Gamma$. The spectrum of a graph $\Gamma$ of its adjacency matrix $\boldsymbol{A}\left(=\boldsymbol{A}_{1}\right)$ is denoted by

$$
\operatorname{sp} \Gamma=\operatorname{sp} \boldsymbol{A}=\left\{\theta_{0}^{m\left(\theta_{0}\right)}, \theta_{1}^{m\left(\theta_{1}\right)}, \ldots, \theta_{d}^{m\left(\theta_{d}\right)}\right\},
$$

where the different eigenvalues of $\Gamma$, whose set is denoted by ev $\Gamma$, are in decreasing order, $\theta_{0}>\theta_{1}>\cdots>\theta_{d}$, and the superscripts stand for their multiplicities $m\left(\theta_{i}\right)$, for $i=0, \ldots, d$. In particular, note that $m\left(\theta_{0}\right)=1$, since $\Gamma$ is connected, and $m\left(\theta_{0}\right)+m\left(\theta_{1}\right)+\cdots+m\left(\theta_{d}\right)=n$. Alternatively, if we include repetitions, the eigenvalues of $\Gamma$ are denoted by $\lambda_{1} \geq \lambda_{2} \geq \cdots \geq \lambda_{n}$.

1.2. Projections and local spectra. For any graph with eigenvalue $\theta_{i}$ having multiplicity $m\left(\theta_{i}\right)$, its corresponding (principal) idempotent can be computed as $\boldsymbol{E}_{i}=\boldsymbol{V}_{i} \boldsymbol{V}_{i}^{\top}$, where $\boldsymbol{V}_{i}$ is the $n \times m\left(\theta_{i}\right)$ matrix whose columns form an orthonormal basis of the eigenspace $\mathcal{E}_{i}=\operatorname{Ker}\left(\boldsymbol{A}-\theta_{i} \boldsymbol{I}\right)$. For instance, when $\Gamma$ is a $\delta$-regular graph on $n$ vertices, its largest eigenvalue $\theta_{0}=\delta$ has eigenvector $\boldsymbol{j}$, the all-1 (column) vector, and corresponding idempotent $\boldsymbol{E}_{0}=\frac{1}{n} \boldsymbol{j} \boldsymbol{j}^{\top}=\frac{1}{n} \boldsymbol{J}$, where $\boldsymbol{J}$ is the all-1 matrix. 
Alternatively, for every $i=0,1, \ldots, d$, the orthogonal projection of $\mathbb{R}^{n}$ onto the eigenspace $\mathcal{E}_{i}$ is given by the Lagrange interpolating polynomial

$$
L_{i}(x)=\frac{1}{\phi_{i}} \prod_{\substack{j=0 \\ j \neq i}}^{d}\left(x-\theta_{j}\right)
$$

of degree $d$, where $\phi_{i}=\prod_{j=0, j \neq i}^{d}\left(\theta_{i}-\theta_{j}\right)$. These polynomials satisfy $L_{i}\left(\theta_{i}\right)=1$ and $L_{i}\left(\theta_{j}\right)=0$ for $j \neq i$. The idempotents are, then,

$$
\boldsymbol{E}_{i}=L_{i}(\boldsymbol{A})=\frac{1}{\phi_{i}} \prod_{\substack{j=0 \\ j \neq i}}^{d}\left(\boldsymbol{A}-\theta_{j} \boldsymbol{I}\right)
$$

and they are known to satisfy the following properties (see, for instance, Godsil [21, p. 28]):

(a) $\boldsymbol{E}_{i} \boldsymbol{E}_{j}=\delta_{i j} \boldsymbol{E}_{i}$

(b) $\boldsymbol{A} \boldsymbol{E}_{i}=\theta_{i} \boldsymbol{E}_{i}$

(c) $p(\boldsymbol{A})=\sum_{i=0}^{d} p\left(\theta_{i}\right) \boldsymbol{E}_{i}$, for any polynomial $p(x) \in \mathbb{R}[x]$.

In particular, when $p(x)=x$ in $(c)$, we have the so-called spectral decomposition theorem: $\boldsymbol{A}=\sum_{i=0}^{d} \theta_{i} \boldsymbol{E}_{i}$. The (u-) local multiplicities of the eigenvalue $\theta_{i}$, introduced by Fiol and Garriga in [17], were defined as the square norm of the projection of $\boldsymbol{e}_{u}$ onto the eigenspace $\mathcal{E}_{i}$, where $\boldsymbol{e}_{u}$ is the unitary characteristic vector of a vertex $u \in V$. That is,

$$
m_{u}\left(\theta_{i}\right)=\left\|\boldsymbol{E}_{i} \boldsymbol{e}_{u}\right\|^{2}=\left\langle\boldsymbol{E}_{i} \boldsymbol{e}_{u}, \boldsymbol{e}_{u}\right\rangle=\left(\boldsymbol{E}_{i}\right)_{u u}, \quad u \in V, \quad i=0,1, \ldots, d
$$

Notice that, in fact, $m_{u}\left(\theta_{i}\right)=\cos ^{2} \beta_{u i}$, where $\beta_{u i}$ is the angle between $\boldsymbol{e}_{u}$ and $\boldsymbol{E}_{i} \boldsymbol{e}_{u}$. The values cos $\beta_{u i}$, for $u \in V$ and $i=0, \ldots, d$, were formally introduced by Cvetković as the 'angles' of $\Gamma$ (see, for instance, Cvetković and Doob [5]).

The local multiplicities can be seen as a generalization of the (standard) multiplicities when the graph is 'seen' from the 'base vertex' $u$. Indeed, they satisfy the following properties (see Fiol and Garriga [17]):

$$
\begin{aligned}
& \sum_{i=0}^{d} m_{u}\left(\theta_{i}\right)=1 \\
& \sum_{u \in V} m_{u}\left(\theta_{i}\right)=m\left(\theta_{i}\right), \quad i=0,1, \ldots, d .
\end{aligned}
$$

If $\mu_{0}\left(=\theta_{0}\right)>\mu_{1}>\cdots>\mu_{d_{u}}$ represent the eigenvalues of $\Gamma$ with non-null $u$-local multiplicity, we define the $u$-local spectrum of $\Gamma$ as

$$
\operatorname{sp}_{u} \Gamma=\left\{\mu_{0}^{m_{u}\left(\mu_{0}\right)}, \mu_{1}^{m_{u}\left(\mu_{1}\right)}, \ldots, \mu_{d_{u}}^{m_{u}\left(\mu_{d_{u}}\right)}\right\}
$$

By analogy with the local multiplicities, which correspond to the diagonal entries of the idempotents, Fiol, Garriga, and Yebra [19] defined the crossed (uv-)local multiplicities of the eigenvalue $\theta_{i}$, denoted by $m_{u v}\left(\theta_{i}\right)$, as

$$
m_{u v}\left(\theta_{i}\right)=\left\langle\boldsymbol{E}_{i} \boldsymbol{e}_{u}, \boldsymbol{E}_{i} \boldsymbol{e}_{v}\right\rangle=\left\langle\boldsymbol{E}_{i} \boldsymbol{e}_{u}, \boldsymbol{e}_{v}\right\rangle=\left(\boldsymbol{E}_{i}\right)_{u v}, \quad u, v \in V, \quad i=0,1, \ldots, d
$$


449 A General Method to Obtain the Spectrum and Local Spectra of a Graph from its Regular Partitions

(Thus, in particular, $m_{u u}\left(\theta_{i}\right)=m_{u}\left(\theta_{i}\right)$.) These parameters allow us to compute the number of walks of length $\ell$ between two vertices $u, v$ in the following way:

$$
a_{u v}^{(\ell)}=\left(\boldsymbol{A}^{\ell}\right)_{u v}=\sum_{i=0}^{d} m_{u v}\left(\theta_{i}\right) \theta_{i}^{\ell}, \quad \ell=0,1, \ldots
$$

Conversely, the values $a_{u v}^{(\ell)}$, for $\ell=0,1, \ldots, d$, determine the crossed local multiplicities $m_{u v}\left(\theta_{i}\right)$. Notice that the coefficients of the system in (1.5) are the entries of a Vandermonde matrix, see, for example, Macon and Spitzbart [26].

2. Regular partitions and local spectra of vertices. Let $\Gamma=(V, E)$ be a graph with adjacency matrix $\boldsymbol{A}$. A partition $\pi=\left(V_{1}, \ldots, V_{m}\right)$ of its vertex set $V$ is called regular (or equitable) whenever, for any $i, j=1, \ldots, m$, the intersection numbers $b_{i j}(u)=\left|\Gamma(u) \cap V_{j}\right|$, where $u \in V_{i}$, do not depend on the vertex $u$ but only on the subsets (usually called classes or cells) $V_{i}$ and $V_{j}$. In this case, such numbers are simply written as $b_{i j}$, and the $m \times m$ matrix $\boldsymbol{B}=\left(b_{i j}\right)$ is referred to as the quotient matrix of $\boldsymbol{A}$ with respect to $\pi$. This is also represented by the quotient (weighted) graph $\pi(\Gamma)$ (associated with the partition $\pi$ ), with vertices representing the cells, and there is an edge with weight $b_{i j}$ between vertex $V_{i}$ and vertex $V_{j}$ if and only if $b_{i j} \neq 0$. Of course, if $b_{i i}>0$, for some $i=1, \ldots, m$, the quotient graph $\pi(\Gamma)$ has loops.

The characteristic matrix of (any) partition $\pi$ is the $n \times m$ matrix $S=\left(s_{u i}\right)$ whose $i$-th column is the characteristic vector of $V_{i}$, that is, $s_{u i}=1$ if $u \in V_{i}$, and $s_{u i}=0$ otherwise. In terms of this matrix, we have the following characterization of regular partitions (see Godsil [21]).

LEMma 2.1. ([21]) Let $\Gamma=(V, E)$ be a graph with adjacency matrix $\boldsymbol{A}$, and vertex partition $\pi$ with characteristic matrix $\boldsymbol{S}$. Then, $\pi$ is regular if and only if there exists an $m \times m$ matrix $\boldsymbol{C}$ such that $\boldsymbol{S C}=\boldsymbol{A} \boldsymbol{S}$. Moreover, $\boldsymbol{C}=\boldsymbol{B}$, the quotient matrix of $\boldsymbol{A}$ with respect to $\pi$.

Using the above lemma, it can be proved that $\operatorname{sp} \boldsymbol{B} \subseteq \operatorname{sp} \boldsymbol{A}$. Moreover, we have the following result by the authors [9].

LEMMA 2.2. ([9]) Let $\Gamma$ be a graph with adjacency matrix $\boldsymbol{A}$. Let $\pi=\left(V_{1}, \ldots, V_{m}\right)$ be a regular partition of $\Gamma$, with quotient matrix $\boldsymbol{B}$. Then, the number of $\ell$-walks from any vertex $u \in V_{i}$ to all vertices of $V_{j}$ is the $i j$-entry $b_{i j}^{(\ell)}$ of $\boldsymbol{B}^{\ell}$.

Now we have the next result, which can be seen as a special case of Lemma 2.2 (see Godsil [21, Cor. 3.3] for the case when $\left.V_{1}=V_{j}=\{u\}\right)$.

LEMMA 2.3. Let $\Gamma$ be a graph with adjacency matrix $\boldsymbol{A}$, and $\pi=\left(V_{1}, \ldots, V_{m}\right)$ a regular partition of $\Gamma$ with quotient matrix $\boldsymbol{B}$. If $V_{1}=\{u\}$, then the number of $\ell$-walks from vertex $u$ to a vertex $v \in V_{j}$, for $j=1, \ldots, m$, only depends on $j$ :

$$
\left(\boldsymbol{A}^{\ell}\right)_{u v}=a_{j}^{(\ell)}=\frac{1}{\left|V_{j}\right|}\left(\boldsymbol{B}^{\ell}\right)_{1 j} .
$$

Proof. To prove that the number of $\ell$-walks between $u$ and $v \in V_{j}$ is a constant, we use induction on $\ell$. The result is clearly true for $\ell=0$, since $\boldsymbol{B}^{0}=\boldsymbol{I}$, and for $\ell=1$ because of the definition of $\boldsymbol{B}$. Suppose that the result holds for some $\ell>1$. Then, the set of walks of length $\ell+1$ from $u$ to $v \in V_{j}$ is obtained from the set of $\ell$-walks from $u$ to vertices $w \in V_{h}$ adjacent to $v$. Then, the number of these walks is

$$
\left(\boldsymbol{A}^{\ell+1}\right)_{u v}=\sum_{h=1}^{m} \sum_{w \in \Gamma(v) \cap V_{h}}\left(\boldsymbol{A}^{\ell}\right)_{u w}=\sum_{h=1}^{m} b_{j h} a_{h}^{(\ell)}=a_{j}^{(\ell+1)}
$$


as claimed.

Alternatively, a more direct proof is to realize that, by Lemma 2.2, we can compute in two ways the number of $\ell$-walks between (the vertices of) $V_{i}$ and $V_{j}$. Namely, $\left|V_{i}\right|\left(\boldsymbol{B}^{\ell}\right)_{i j}=\left|V_{j}\right|\left(\boldsymbol{B}^{\ell}\right)_{j i}$ and, since this holds for any $\ell \geq 0$,

$$
\left|V_{i}\right|(p(\boldsymbol{B}))_{i j}=\left|V_{j}\right|(p(\boldsymbol{B}))_{j i}
$$

for any polynomial $p(x)$. Thus, in the lemma, we take the special case $V_{i}=V_{1}=\{u\}$ and $p(x)=x^{\ell}$ (notice that $\left(\boldsymbol{B}^{\ell}\right)_{j 1}=\left(\boldsymbol{A}^{\ell}\right)_{u v}$ for $\left.v \in V_{j}\right)$.

Let $\boldsymbol{B}$ be a quotient (diagonalizable) $m \times m$ matrix as above, with sp $\boldsymbol{B}=\left\{\tau_{0}^{m\left(\tau_{0}\right)}, \tau_{1}^{m\left(\tau_{1}\right)}, \ldots, \tau_{e}^{m\left(\tau_{e}\right)}\right\}$, and let $\boldsymbol{D}$ be the diagonal matrix with entries the eigenvalues of $\boldsymbol{B}$ (including repetitions). Let $\boldsymbol{Q}$ be the $m \times m$ matrix that diagonalizes $\boldsymbol{B}$, that is, $\boldsymbol{Q}^{-1} \boldsymbol{B} \boldsymbol{Q}=\boldsymbol{D}$. For $i=0, \ldots, e$, let $\boldsymbol{V}_{i}$ be the $m \times m\left(\tau_{i}\right)$ matrix formed by the columns of $\boldsymbol{Q}$ corresponding to the right $\tau_{i}$-eigenvectors of $\boldsymbol{B}$. Let $\boldsymbol{U}_{i}$ be the $m\left(\tau_{i}\right) \times m$ matrix formed by the corresponding rows of $\boldsymbol{Q}^{-1}$, which are the left $\tau_{i}$-eigenvectors of $\boldsymbol{B}$. Then, the $i$-th idempotent of $\boldsymbol{B}$ is $\overline{\boldsymbol{E}}_{i}=\boldsymbol{V}_{i} \boldsymbol{U}_{i}$. Moreover, $\overline{\boldsymbol{E}}_{i}$ can be computed as in the case of the (symmetric) adjacency matrix by using the Lagrange interpolating polynomial $\bar{L}_{i}(x)$ satisfying $\bar{L}_{i}\left(\tau_{j}\right)=\delta_{i j}$ :

$$
\overline{\boldsymbol{E}}_{i}=\bar{L}_{i}(\boldsymbol{B})=\frac{1}{\prod_{\substack{j=0 \\ j \neq i}}^{e}\left(\tau_{i}-\tau_{j}\right)} \prod_{\substack{j=0 \\ j \neq i}}^{e}\left(\boldsymbol{B}-\tau_{j} \boldsymbol{I}\right)
$$

The above results yield a simple method to compute the local spectra of a vertex $u$ in a given regular partition or, more generally, the crossed multiplicities between $u$ and any other vertex $v$. Besides, with the union of the local spectra (applying (1.4)) of the different classes of vertices according to their corresponding regular partitions (that is, we 'hang' the quotient graph from every one of the different classes of vertices), for the very first time all the spectrum of the original graph is obtained from regular partitions. Thus, the main result is the following.

TheOrem 2.4. Let $\Gamma$ be a graph with adjacency matrix $\boldsymbol{A}$ and set of different eigenvalues $\mathrm{ev} \Gamma=$ $\left\{\theta_{0}, \theta_{1}, \ldots, \theta_{d}\right\}$. Let $\pi=\left(V_{1}, \ldots, V_{m}\right)$ be a regular partition of $\Gamma$, with $V_{1}=\{u\}$. Let $\boldsymbol{B}$ be the quotient matrix of $\pi$, with set of different eigenvalues ev $\boldsymbol{B}=\left\{\tau_{0}, \tau_{1}, \ldots, \tau_{e}\right\} \subseteq$ ev $\Gamma$. Let $L_{i}(x)$ and $\bar{L}_{i}(x)$ be the Lagrange interpolating polynomials satisfying $L_{i}\left(\theta_{j}\right)=\delta_{i j}$ for $i, j=0, \ldots, d$, and $\bar{L}_{i}\left(\tau_{j}\right)=\delta_{i j}$ for $i, j=0, \ldots, e$, respectively. Let $\boldsymbol{E}_{i}=L_{i}(\boldsymbol{A})$ and $\overline{\boldsymbol{E}}_{i}=\bar{L}_{i}(\boldsymbol{B})$ be the corresponding idempotents. Then, for every vertex $v \in V_{j}$, the crossed uv-local multiplicity of $\theta_{i}$ is

$$
m_{u v}\left(\theta_{i}\right)=\frac{1}{\left|V_{j}\right|}\left(L_{i}(\boldsymbol{B})\right)_{1 j}, \quad i=0,1, \ldots, d,
$$

or, alternatively,

$$
m_{u v}\left(\theta_{i}\right)=\left\{\begin{array}{cc}
\frac{1}{\left|V_{j}\right|}\left(\overline{\boldsymbol{E}}_{i}\right)_{1 j} & \text { if } \theta_{i} \in \mathrm{ev} \boldsymbol{B} \\
0 & \text { otherwise }
\end{array}\right.
$$

Proof. Let $L_{i}(x)=\sum_{r=0}^{d} \zeta_{r} x^{r}$. Then, for every $v \in V_{j}$ and $i=0,1, \ldots, d$, and using Lemma 2.3, we 
451 A General Method to Obtain the Spectrum and Local Spectra of a Graph from its Regular Partitions

have

$$
\begin{aligned}
m_{u v}\left(\theta_{i}\right) & =\left(\boldsymbol{E}_{i}\right)_{u v}=\left(L_{i}(\boldsymbol{A})\right)_{u v}=\sum_{r=0}^{d} \zeta_{r}\left(\boldsymbol{A}^{r}\right)_{u v} \\
& =\frac{1}{\left|V_{j}\right|} \sum_{r=0}^{d} \zeta_{r}\left(\boldsymbol{B}^{r}\right)_{1 j}=\frac{1}{\left|V_{j}\right|}\left(L_{i}(\boldsymbol{B})\right)_{1 j},
\end{aligned}
$$

which proves (2.8). To prove (2.9), note first that, by the spectral decomposition theorem, $\boldsymbol{B}^{r}=\sum_{i=0}^{e} \tau_{i}^{r} \overline{\boldsymbol{E}}_{i}$. Then, by Lemma 2.3, the numbers of $\ell$-walks from $u$ to $v \in V_{j}$ are

$$
a_{u v}^{(\ell)}=\frac{1}{\left|V_{j}\right|} \sum_{i=0}^{e} \tau_{i}^{\ell}\left(\overline{\boldsymbol{E}}_{i}\right)_{1 j}, \quad \ell=0, \ldots, d
$$

which, as already commented, determine the local multiplicities because of the system of equations

$$
\sum_{i=0}^{d} \theta_{i}^{\ell} m_{u v}\left(\theta_{i}\right)=a_{u v}^{(\ell)}, \quad \ell=0, \ldots, d
$$

But a (the) solution of this system is obtained when the multiplicities $m_{u v}\left(\theta_{i}\right)$, for $i=0, \ldots, d$, are given by (2.9), as claimed.

In particular, notice that this result allows us to compute the $u$-local spectrum of $\Gamma$ as

$$
\operatorname{sp}_{u} \Gamma=\left\{\tau_{0}^{m_{u}\left(\tau_{0}\right)}, \tau_{1}^{m_{u}\left(\tau_{1}\right)}, \ldots, \tau_{e}^{m_{u}\left(\tau_{e}\right)}\right\}
$$

where $\tau_{i} \in$ ev $\boldsymbol{B}$ and $m_{u}\left(\tau_{i}\right)=\left(\overline{\boldsymbol{E}}_{i}\right)_{11}$, for $i=0, \ldots, e$.

Let us show an example.

ExAmPLE 2.5. Let $\Delta=\Gamma+u$ be the cone of a $k$-regular graph on $n$ vertices, where the 'new' vertex $u$ is joined to all vertices of $\Gamma$. Then, $\Delta$ has a regular partition with quotient matrix

$$
\boldsymbol{B}=\left(\begin{array}{ll}
0 & n \\
1 & k
\end{array}\right)
$$

and eigenvalues $\theta_{0}=\frac{1}{2}\left(k+\sqrt{k^{2}+4 n}\right)$ and $\theta_{1}=\frac{1}{2}\left(k-\sqrt{k^{2}+4 n}\right)$. (Notice that the first expression can be rewritten as $k=\theta_{0}-\frac{n}{\theta_{0}}$, in agreement with the results of Dalfó, Fiol, and Garriga [10].) Thus, the idempotents of $\boldsymbol{B}$ turn out to be $\overline{\boldsymbol{E}}_{0}=\left(\boldsymbol{B}-\theta_{1} \boldsymbol{I}\right) /\left(\theta_{0}-\theta_{1}\right)$ and $\overline{\boldsymbol{E}}_{1}=\left(\boldsymbol{B}-\theta_{0} \boldsymbol{I}\right) /\left(\theta_{1}-\theta_{0}\right)$. Consequently, Theorem 2.4 implies that the local $u$-spectrum of $\Delta$ is

$$
\operatorname{sp}_{u} \Delta=\left\{\frac{1}{2}\left(k+\sqrt{k^{2}+4 n}\right)^{m_{u}\left(\theta_{0}\right)}, \frac{1}{2}\left(k-\sqrt{k^{2}+4 n}\right)^{m_{u}\left(\theta_{1}\right)}\right\},
$$

where $m_{u}\left(\theta_{0}\right)=\frac{1}{2}\left(1-k / \sqrt{k^{2}+4 n}\right)$ and $m_{u}\left(\theta_{1}\right)=\frac{1}{2}\left(1+k / \sqrt{k^{2}+4 n}\right)$.

Another simple consequence of our main result is obtained for simple eigenvalues of $\boldsymbol{B}$.

Corollary 2.6. Let $\Gamma$ be a graph with adjacency matrix $\boldsymbol{A}$ and a set of different eigenvalues ev $\Gamma=$ $\left\{\theta_{0}, \theta_{1}, \ldots, \theta_{d}\right\}, \pi=\left(V_{1}, \ldots, V_{m}\right)$ a regular partition of $\Gamma$ with $V_{1}=\{u\}, \boldsymbol{B}$ the quotient matrix of $\pi$ with 
a set of different eigenvalues ev $\boldsymbol{B}=\left\{\tau_{0}, \tau_{1}, \ldots, \tau_{e}\right\} \subseteq$ ev $\Gamma$, and $\boldsymbol{E}_{i}$ and $\overline{\boldsymbol{E}}_{i}$ the corresponding idempotents. Suppose that, for some $i, \theta_{i} \in$ ev $\boldsymbol{A} \cap$ ev $\boldsymbol{B}$ has multiplicity 1 . Let $\boldsymbol{u}_{i}=\left(u_{i 1}, \ldots, u_{i m}\right)$ and $\boldsymbol{v}_{i}=\left(v_{1 i}, \ldots, v_{i m}\right)^{\top}$ be the left and right eigenvectors of $\boldsymbol{B}$, respectively, corresponding to the eigenvalue $\theta_{i}$. Then, for every vertex $v \in V_{j}$, the crossed uv-local multiplicity of $\theta_{i}$ in $\Gamma$ is

$$
m_{u v}\left(\theta_{i}\right)=\frac{1}{\left|V_{j}\right|} \frac{v_{1 i} u_{i j}}{\left\langle\boldsymbol{u}_{i}, \boldsymbol{v}_{i}\right\rangle}, \quad j=1, \ldots, m .
$$

Proof. Let $\boldsymbol{Q}$ be a matrix that diagonalizes $\boldsymbol{B}$. If, for some constants $\alpha$ and $\beta$, we have that $\alpha \boldsymbol{u}_{i}$ and $\beta \boldsymbol{v}_{i}$ are the corresponding row of $\boldsymbol{Q}^{-1}$ and column of $\boldsymbol{Q}$, respectively, then $\left(\boldsymbol{Q}^{-1} \boldsymbol{Q}\right)_{i i}=1$ implies that $\alpha \beta=\left\langle\boldsymbol{u}_{i}, \boldsymbol{v}_{i}\right\rangle^{-1}$. Thus, $\left(\bar{E}_{i}\right)_{1 j}=\left(\alpha \boldsymbol{v}_{i} \cdot \beta \boldsymbol{u}_{i}\right)_{1 j}=\frac{v_{1 i} u_{i j}}{\left\langle\boldsymbol{u}_{i}, \boldsymbol{v}_{i}\right\rangle}$ (where ''' stands for the matrix product), and the result follows from $(2.9)$.

Alternatively, if $\boldsymbol{u}_{i}$ and $\boldsymbol{v}_{i}$ are already taken from the corresponding row and column of $\boldsymbol{Q}^{-1}$ and $\boldsymbol{Q}$, respectively, then $\alpha=\beta=1$, and (2.11) can be simply written as

$$
m_{u v}\left(\tau_{i}\right)=\frac{1}{\left|V_{j}\right|}\left(\boldsymbol{Q}^{-1}\right)_{i j}(\boldsymbol{Q})_{1 i}, \quad j=1, \ldots, m
$$

3. Regular partitions and local spectra of vertex sets. Let $\Gamma$ be a graph with vertex set $V$. The results of the last section can be generalized to find the so-called local multiplicities of a vertex subset $C \subset V$. In this case, we consider the normalized characteristic vector

$$
e_{C}=\frac{1}{\sqrt{|C|}} \sum_{u \in C} e_{u}
$$

Then, the $C$-local multiplicity of the eigenvalue $\theta_{i}$ is defined as

$$
\begin{aligned}
m_{C}\left(\theta_{i}\right) & =\left\|\boldsymbol{E}_{i} \boldsymbol{e}_{C}\right\|^{2}=\left\langle\boldsymbol{E}_{i} \boldsymbol{e}_{C}, \boldsymbol{e}_{C}\right\rangle=\frac{1}{|C|} \sum_{u, v \in C}\left\langle\boldsymbol{E}_{i} \boldsymbol{e}_{u}, \boldsymbol{e}_{v}\right\rangle \\
& =\frac{1}{|C|} \sum_{u, v \in C} m_{u v}\left(\theta_{i}\right), \quad i=0,1, \ldots, d
\end{aligned}
$$

Note that the sequence of $C$-local multiplicities $m_{C}\left(\theta_{0}\right), \ldots, m_{C}\left(\theta_{d}\right)$ is, in fact, the so-called Mac Williams transform of the vector $\boldsymbol{e}_{C}$; see, for instance, Delsarte and Levenshtein [14]. Observe that, since $\boldsymbol{e}_{C}$ is a unit vector, we have $\sum_{i=0}^{d} m_{C}\left(\theta_{i}\right)=1$. Moreover, for $C \neq \emptyset$, the $C$-local multiplicity of $\theta_{0}$ is $m_{C}\left(\theta_{0}\right)=|C| /|V|>$ 0 .

As expected, the $C$-local multiplicities are relevant when studying the graph from the vertex subset $C$. For instance, the number $a_{C C}^{(\ell)}$ of walks of length $\ell$ from (the vertices of) $C$ to itself is given by

$$
a_{C C}^{(\ell)}=\sum_{u, v}\left(\boldsymbol{A}^{\ell}\right)_{u v}=|C|\left\langle\boldsymbol{A}^{\ell} \boldsymbol{e}_{C}, \boldsymbol{e}_{C}\right\rangle=|C| \sum_{i=0}^{d} m_{C}\left(\theta_{i}\right) \theta_{i}^{\ell} .
$$

If $\mu_{0}\left(=\theta_{0}\right)>\mu_{1}>\cdots>\mu_{d_{C}}$ represent the eigenvalues of $\Gamma$ with nonzero $C$-local multiplicities, then the $C$-local spectrum of $\Gamma$ is

$$
\operatorname{sp}_{C} \Gamma=\left\{\mu_{0}^{m_{C}\left(\mu_{0}\right)}, \mu_{1}^{m_{C}\left(\mu_{1}\right)}, \ldots, \mu_{d_{C}}^{m_{C}\left(\mu_{d_{C}}\right)}\right\}
$$


453 A General Method to Obtain the Spectrum and Local Spectra of a Graph from its Regular Partitions

For more details about $C$-local multiplicities and their applications, see Section 3.1 or Cámara, Fàbrega, Fiol, and Garriga [4].

In our context of having a regular partition, we have the following result.

TheOREM 3.1. Let $\Gamma$ be a graph with adjacency matrix $\boldsymbol{A}$ and set of different eigenvalues ev $\Gamma=$ $\left\{\theta_{0}, \theta_{1}, \ldots, \theta_{d}\right\}$. Let $\pi=\left(V_{1}, \ldots, V_{m}\right)$ be a regular partition of $\Gamma$. Let $\boldsymbol{B}$ be the quotient matrix of $\pi$, with set of different eigenvalues ev $\boldsymbol{B}=\left\{\mu_{0}, \mu_{1}, \ldots, \mu_{e}\right\} \subseteq$ ev $\Gamma$. Let $L_{i}(x)$ and $\bar{L}_{i}(x)$ be the Lagrange interpolating polynomials satisfying $L_{i}\left(\theta_{j}\right)=\delta_{i j}$ for $i, j=0, \ldots, d$ and $\bar{L}_{i}\left(\mu_{j}\right)=\delta_{i j}$ for $i, j=0, \ldots$, , respectively. Let $\boldsymbol{E}_{i}=L_{i}(\boldsymbol{A})$ and $\overline{\boldsymbol{E}}_{i}=\bar{L}_{i}(\boldsymbol{B})$ be the corresponding idempotents. Then, for every class $V_{j}$, the $V_{j}$-local multiplicity of $\theta_{i}$ is

$$
m_{V_{j}}\left(\theta_{i}\right)=\left(L_{i}(\boldsymbol{B})\right)_{j j}, \quad i=0,1, \ldots, d
$$

or, alternatively,

$$
m_{V_{j}}\left(\theta_{i}\right)=\left\{\begin{array}{cc}
\left(\overline{\boldsymbol{E}}_{i}\right)_{j j} & \text { if } \theta_{i} \in \mathrm{ev} \boldsymbol{B} \\
0 & \text { otherwise }
\end{array}\right.
$$

Proof. An immediate and known consequence of Lemma 2.1 is that, for any polynomial $p(x)$,

$$
p(\boldsymbol{A}) \boldsymbol{S}=\boldsymbol{S} p(\boldsymbol{B}) .
$$

(In fact, multiplying both terms by $\boldsymbol{S}^{\top}$, and taking into account that both resulting matrices are symmetric, we can prove again (2.7)). Then, with $p(x)=L_{i}(x)$ in (3.17), we get $\boldsymbol{E}_{i} \boldsymbol{S}=\boldsymbol{S} L_{i}(\boldsymbol{B})$, whence $\boldsymbol{S}^{\top} \boldsymbol{E}_{i} \boldsymbol{S}=$ $\boldsymbol{S}^{\top} \boldsymbol{S} L_{i}(\boldsymbol{B})=\boldsymbol{D} L_{i}(\boldsymbol{B})$, where $\boldsymbol{D}=\operatorname{diag}\left(\left|V_{1}\right|, \ldots,\left|V_{m}\right|\right)$ and, using (3.13),

$$
\begin{aligned}
m_{V_{j}}\left(\theta_{i}\right) & =\frac{1}{\left|V_{j}\right|} \sum_{u, v \in V_{j}} m_{u v}\left(\theta_{i}\right)=\frac{1}{\left|V_{j}\right|}\left(\boldsymbol{S}^{\top} \boldsymbol{E}_{i} \boldsymbol{S}\right)_{j j} \\
& =\frac{1}{\left|V_{j}\right|}(\boldsymbol{D})_{j j}\left(L_{i}(\boldsymbol{B})\right)_{j j}=\left(L_{i}(\boldsymbol{B})\right)_{j j}, \quad i=0,1, \ldots, d,
\end{aligned}
$$

which proves (3.15). The proof of (3.16) goes similarly as that of (2.9) by using $\boldsymbol{B}^{\ell}=\sum_{i=0}^{e} \mu_{i}^{\ell} \overline{\boldsymbol{E}}_{i}$ and $a_{V_{i} V_{i}}^{(\ell)}=\left|V_{i}\right|\left(\boldsymbol{B}^{\ell}\right)_{i i}$ by Lemma 2.2 .

Let us see a simple example.

EXAMPLE 3.2. Let $\Gamma=(V, E)$ be a $k$-regular graph on $n$ vertices. Let $C \subset V$ be a 1 -perfect code in $\Gamma$ (where any vertex not in $C$ is adjacent to exactly one vertex of $C$; see for instance Godsil [21]). Then, $|C|=n /(1+k)$, and the partition $V=C \cup \bar{C}$ is regular with quotient matrix

$$
\boldsymbol{B}=\left(\begin{array}{cc}
0 & k \\
1 & k-1
\end{array}\right)
$$

whose eigenvalues are $\mu_{0}=k$ and $\mu_{1}=-1$. Then, the idempotents of $\boldsymbol{B}$ turn out to be $\overline{\boldsymbol{E}}_{0}=(\boldsymbol{B}+\boldsymbol{I}) /(k+1)$ and $\overline{\boldsymbol{E}}_{1}=(\boldsymbol{B}-k \boldsymbol{I}) /(-1-k)$. Consequently, Theorem 3.1 implies that the $C$-local and $\bar{C}$-local multiplicities of $\Gamma$ are

and

$$
m_{C}(k)=\frac{1}{k+1}(=|C| /|V|), \quad m_{C}(-1)=\frac{k}{k+1},
$$

$$
m_{\bar{C}}(k)=\frac{k}{k+1}(=|\bar{C}| /|V|), \quad m_{\bar{C}}(-1)=\frac{k}{k+1} .
$$


3.1. The $C$-local spectra and completely regular codes. The last example is a particular case of a completely regular code. As a generalization of this example, now we describe some applications of the local spectrum of a vertex subset $C$ in the characterization of such codes. This gives a further justification for our results in this section.

As above, let $C \subset V$ be a vertex subset of a graph $\Gamma$. The distance from $C$ to a given vertex $u$ of $V$ is given by $\operatorname{dist}(C, u)=\min _{v \in C}\{\operatorname{dist}(u, v)\}$. This definition gives us a distance partition of $V$ where each part $C_{k}$ is the set of vertices at distance $k$ from $C$. It is natural to say that the eccentricity of $C$ is given by $\varepsilon_{C}=\max _{u \in V}\{\operatorname{dist}(C, u)\}$, and it is known that if $C$ has $d_{C}+1$ distinct $C$-local values, then $\varepsilon_{C} \leq d_{C}$.

We say that $G$ is distance-regular around $C$, with eccentricity $\varepsilon=\operatorname{ecc}(C)$, if the distance partition $V=C_{0} \cup C_{1} \cup \cdots \cup C_{\varepsilon}$ is regular, The set $C$ is also referred to as a completely regular set or completely regular code (see Godsil [21]). In [18] Fiol and Garriga gave the following characterization of completely regular codes in terms of the number of vertices at (spectrally maximum) distance $d_{C}$ from $C$.

Theorem $3.3([18])$. Let $\Gamma=(V, E)$ be a regular graph on $n$ vertices. A vertex subset $C \subset V$, with $r$ vertices and local spectrum $\operatorname{sp}_{C} \Gamma=\left\{\mu_{0}^{m_{C}\left(\mu_{0}\right)}, \mu_{1}^{m_{C}\left(\mu_{1}\right)}, \ldots, \mu_{d_{C}}^{m_{C}\left(\mu_{d_{C}}\right)}\right\}$, is a completely regular code if and only if the number of vertices at distance $d_{C}$ from $C$ satisfies

$$
\left|C_{d_{C}}\right|=\frac{n^{2}}{r}\left(\sum_{i=0}^{d_{C}} \frac{\pi_{0}^{2}}{m_{C}\left(\mu_{i}\right) \pi_{i}^{2}}\right)^{-1}
$$

where $\pi_{i}=\prod_{j=0(j \neq i)}^{d}\left|\mu_{i}-\mu_{j}\right|$ for $i=0, \ldots, d_{c}$.

In the case when $\Gamma$ is a distance-regular graph, with diameter $d$ and spectrum as in (1.1), the above result implies that $C$ is a completely regular code if and only if $\left|C_{d_{C}}\right|$ satisfies an expression in terms of the (standard) spectrum of $\Gamma$ and the so-called inner distribution $r_{k}$, for $k=0, \ldots, d$, of $C$. That is, $r_{k}$ is the mean number of vertices $v$ in $C$ at distance $k$ (in $\Gamma$ ) from a given vertex $u \in C$ :

$$
r_{k}=\frac{1}{|C|} \sum_{u \in C}\left|\Gamma_{k}(u) \cap C\right| \text { for } k=0, \ldots, d .
$$

Notice that, as commented by Godsil [21], the numbers $r_{k}$ determine the probability that a randomly chosen pair of vertices from $C$ are at distance $k$. Then, when $\Gamma$ is a distance-regular graph, these numbers are closely related to the $C$-local multiplicities. Indeed, Fiol and Garriga [20] showed that, in this case, the sets of numbers $\left\{r_{0}, \ldots, r_{d}\right\}$ and $\left.\left\{m_{C}\left(\theta_{0}\right), \ldots, m_{C}\left(\theta_{d}\right)\right)\right\}$ are related to each other by the formulas

$$
\begin{aligned}
r_{k} & =\sum_{i=0}^{d} m_{C}\left(\theta_{i}\right) p_{k}\left(\theta_{i}\right) \quad(0 \leq k \leq d), \\
m_{C}\left(\theta_{i}\right) & =\frac{m\left(\theta_{i}\right)}{n} \sum_{k=0}^{d} r_{k} \frac{p_{k}\left(\theta_{i}\right)}{p_{k}\left(\theta_{0}\right)} \quad(0 \leq i \leq d),
\end{aligned}
$$

where $p_{0}, \ldots, p_{d}$ of $\Gamma$ are the distance polynomials of $\Gamma$. Then, by using (3.20), (3.18) becomes

$$
\left|C_{d}\right|=\frac{n}{r}\left(\sum_{i=0}^{d} \frac{\pi_{0}^{2}}{m\left(\theta_{i}\right) \pi_{i}^{2}}\left(\sum_{k=0}^{d} r_{k} \frac{p_{k}\left(\theta_{i}\right)}{p_{k}\left(\theta_{0}\right)}\right)^{-1}\right)^{-1} .
$$


455 A General Method to Obtain the Spectrum and Local Spectra of a Graph from its Regular Partitions

In fact, (3.20) is essentially equivalent to Delsarte's identity $b=a Q$ which gives rise to the celebrated linear programming bound (see Delsarte [13], and Delsarte and Levenshtein [14]), based on the non-negativity of the $C$-local eigenvalues. This produces upper bounds on the size of a code with a given minimum distance, and lower bounds on the size of a design with a given strength, since it gives stronger necessary conditions for their existence. For example, to bound the size of a code $C$ with prescribed parameters $(\epsilon, r, \delta)$ (where $\epsilon$ is the maximum distance between some vertex of $C$ and some vertex of $V, r=|C|$, and $\delta$ is the maximum distance between vertices of $C$ ), we have $r_{1}=\cdots=r_{\delta-1}=0$, and the linear programming problem to be solved is the following:

$$
\begin{gathered}
\text { maximizer }:=1+\sum_{i=\delta}^{d} r_{i} \\
\text { subject to } m_{C}\left(\theta_{j}\right) \geq 0, j=0,1, \ldots, d ; \\
r_{i} \geq 0, i=\delta, \ldots, d
\end{gathered}
$$

The same technique can be applied to deriving results about the existence of disjoint copies of some subgraph of a (putative) distance-regular graph; see, for instance, Dalfó [8].

With respect to bounding the size of a code, some other methods have been proposed; see, for instance, Lovász [25] and Schrijver [28]. In our context, and for the case of completely regular codes, Theorem 3.3, or its particular case of distance-regular graphs (3.21), can be seen as an improvement of Delsarte's linear programming method, or the mentioned methods in [25] and [28]. The reason is that Theorem 3.3 provides a necessary and sufficient condition for the existence of such codes, by using either the $C$-local multiplicities or the inner distribution (in the case of distance-regular graphs). Moreover, in such a case, and as an intermediate step, an application of our method would be to find the $C$-local multiplicities to get the (possible) inner distribution from (3.19).

4. The local spectra of some families of graphs. In this section, we show that the application of our method to obtain the local spectra and the complete spectrum of different well-known families of graphs.

4.1. Walk-regular graphs. Let $\Gamma$ be a graph with spectrum as above. If the number of closed walks of length $\ell$ rooted at vertex $u$, that is, $a_{u u}^{(\ell)}=\sum_{i=0}^{d} m_{u}\left(\theta_{i}\right) \theta_{i}^{\ell}$ only depends on $\ell$, for each $\ell \geq 0$, then $\Gamma$ is called walk-regular (a concept introduced by Godsil and McKay [22]). In this case, we write $a_{u u}^{(\ell)}=a^{(\ell)}$. Note that, since $a_{u u}^{(2)}=\delta(u)$, the degree of vertex $u$, a walk-regular graph is necessarily regular. Moreover, we say that $\Gamma$ is spectrum-regular if, for any $i=0,1, \ldots, d$, the $u$-local multiplicity of $\theta_{i}$ does not depend on the vertex $u$. By (1.5) and the subsequent comment, it follows that spectrum-regularity and walk-regularity are equivalent concepts. Equation (1.5) also shows that the existence of the constants $a^{(0)}, a^{(1)}, \ldots, a^{(d)}$ suffices to assure walk-regularity. It is well known that any distance-regular graph, as well as any vertex-transitive graph, is walk-regular, but the converse is not true. Other particular families of walk-regular graphs are the so-called orbit polynomial graphs, introduced by Beezer [2], and the quotient-polynomial graphs defined in Fiol [16]. The quotient-polynomial graphs could be thought of as the regular counterpart of orbit polynomial graphs. In our context, distance-regular graphs, orbit polynomial graphs, and quotient-polynomial graphs are especially interesting since their structures are described with well-established regular partitions. Moreover, every quotient-polynomial graph generates a (symmetric) association scheme, see again [16].

Proposition 4.1. Let $\Gamma$ be a walk-regular graph with $n$ vertices, having a regular partition $\pi=\left(V_{1}, \ldots\right.$, $\left.V_{m}\right)$ with $V_{1}=\{u\}$ and quotient matrix $\boldsymbol{B}$. Then, the spectrum of $\Gamma$ is

$$
\operatorname{sp} \Gamma=\left\{\theta_{0}^{m\left(\theta_{0}\right)}, \theta_{1}^{m\left(\theta_{1}\right)}, \ldots, \theta_{d}^{m\left(\theta_{d}\right)}\right\}
$$


where, for every $i=0, \ldots, d, \theta_{i}$ also is an eigenvalue of $\boldsymbol{B}$, with multiplicity

$$
m\left(\theta_{i}\right)=n\left(\overline{\boldsymbol{E}}_{i}\right)_{11}=\frac{n}{\prod_{\substack{j=0 \\ j \neq i}}^{d}\left(\theta_{i}-\theta_{j}\right)}\left(\prod_{\substack{j=0 \\ j \neq i}}^{d}\left(\boldsymbol{B}-\theta_{j} \boldsymbol{I}\right)\right)_{11} .
$$

Proof. Since $\sum_{u \in V} m_{u u}\left(\theta_{i}\right)=m\left(\theta_{i}\right)$, the (standard) multiplicity $m\left(\theta_{i}\right)$ 'splits' equitably among the $n$ vertices, giving $m_{u}\left(\theta_{i}\right)=m\left(\theta_{i}\right) / n$. Therefore, the different eigenvalues of $\boldsymbol{B}$ coincide with those of $\boldsymbol{A}$, and Theorem 2.4 yields (4.23).

Let us see an example.

ExAmPle 4.2. Consider the walk-regular graph $\Gamma$, that is not distance-regular, given by Godsil [21]. This graph, and two of its possible quotient graphs, $\pi_{1}(\Gamma)$ (with seven vertices) and $\pi_{2}(\Gamma)$ (with five vertices), are represented in Figure 1. Then, we can obtain the whole spectrum of $\Gamma$ from any of the corresponding regular partitions, $\pi_{1}$ or $\pi_{2}$. For instance, the quotient matrix of $\pi_{2}$ is

$$
\boldsymbol{B}=\left(\begin{array}{lllll}
0 & 4 & 0 & 0 & 0 \\
1 & 1 & 1 & 1 & 0 \\
0 & 2 & 0 & 2 & 0 \\
0 & 1 & 1 & 1 & 1 \\
0 & 0 & 0 & 4 & 0
\end{array}\right)
$$

The spectrum of $\boldsymbol{B}$ related to $\pi_{2}(\Gamma)$ is

$$
\operatorname{sp} \pi_{2}(\Gamma)=\left\{\theta_{0}^{m\left(\tau_{0}\right)}, \theta_{1}^{m\left(\tau_{1}\right)}, \theta_{2}^{m\left(\tau_{2}\right)}, \theta_{3}^{m\left(\tau_{3}\right)}\right\}=\left\{4^{1}, 2^{2}, 0^{1},-2^{3},\right\} .
$$

Since $\Gamma$ is walk-regular, the spectrum of its quotient from a regular partition has all the different eigenvalues of the spectrum of $\Gamma$. Now we compute the multiplicities $m\left(\theta_{i}\right)$, for $i=0,1,2,3$ :

$$
\begin{aligned}
& m\left(\theta_{0}\right)=\frac{12}{\left(\theta_{0}-\theta_{1}\right)\left(\theta_{0}-\theta_{2}\right)\left(\theta_{0}-\theta_{3}\right)}\left(\left(\boldsymbol{B}-\theta_{1} \boldsymbol{I}\right)\left(\boldsymbol{B}-\theta_{2} \boldsymbol{I}\right)\left(\boldsymbol{B}-\theta_{3} \boldsymbol{I}\right)\right)_{11}=1, \\
& m\left(\theta_{1}\right)=\frac{12}{\left(\theta_{1}-\theta_{0}\right)\left(\theta_{1}-\theta_{2}\right)\left(\theta_{1}-\theta_{3}\right)}\left(\left(\boldsymbol{B}-\theta_{0} \boldsymbol{I}\right)\left(\boldsymbol{B}-\theta_{2} \boldsymbol{I}\right)\left(\boldsymbol{B}-\theta_{3} \boldsymbol{I}\right)\right)_{11}=3, \\
& m\left(\theta_{2}\right)=\frac{12}{\left(\theta_{2}-\theta_{0}\right)\left(\theta_{2}-\theta_{1}\right)\left(\theta_{2}-\theta_{3}\right)}\left(\left(\boldsymbol{B}-\theta_{0} \boldsymbol{I}\right)\left(\boldsymbol{B}-\theta_{1} \boldsymbol{I}\right)\left(\boldsymbol{B}-\theta_{3} \boldsymbol{I}\right)\right)_{11}=3, \\
& m\left(\theta_{3}\right)=\frac{12}{\left(\theta_{3}-\theta_{0}\right)\left(\theta_{3}-\theta_{1}\right)\left(\theta_{3}-\theta_{2}\right)}\left(\left(\boldsymbol{B}-\theta_{0} \boldsymbol{I}\right)\left(\boldsymbol{B}-\theta_{1} \boldsymbol{I}\right)\left(\boldsymbol{B}-\theta_{2} \boldsymbol{I}\right)\right)_{11}=5 .
\end{aligned}
$$

This gives that the spectrum of $\Gamma$ is $\operatorname{sp} \Gamma=\left\{4^{1}, 2^{3}, 0^{3},-2^{5}\right\}$, as it is known to be. Note that, in this example, we need to 'hang' the graph $\Gamma$ from only one of its vertices.

4.2. Distance-regular graphs. In particular, when $\Gamma$ is distance-regular, the distance-partition with respect to any vertex is regular with the same quotient matrix $\boldsymbol{B}$ (see, for instance, Biggs [1] or Fiol [15]). Moreover, since $\boldsymbol{B}$ is tridiagonal, all its eigenvalues are simple and (4.23), together with (2.11), leads to the known formula (see Biggs [1] again)

$$
m\left(\theta_{i}\right)=n\left(\overline{\boldsymbol{E}}_{i}\right)_{11}=\frac{n}{\left\langle\boldsymbol{u}_{i}, \boldsymbol{v}_{i}\right\rangle}, \quad j=1, \ldots, m
$$

where the eigenvectors $\boldsymbol{u}_{i}$ and $\boldsymbol{v}_{i}$ have been chosen to have the first entry 1. 
457 A General Method to Obtain the Spectrum and Local Spectra of a Graph from its Regular Partitions

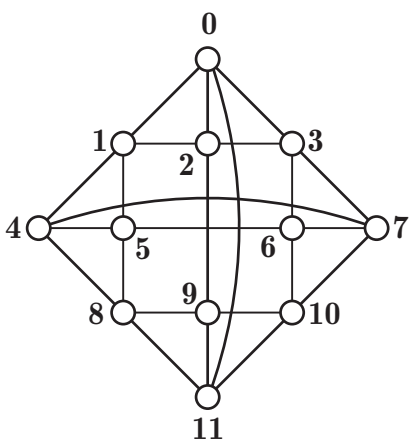

$\Gamma$

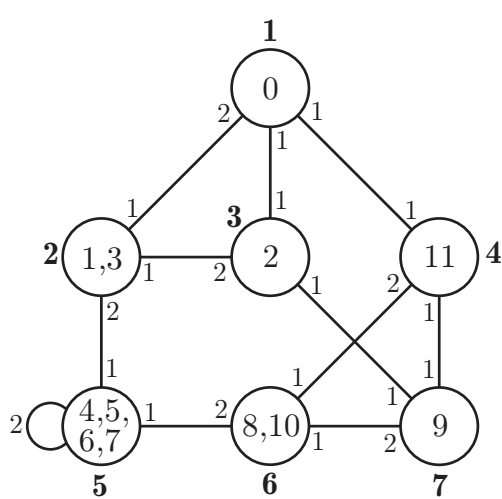

$\pi_{1}(\Gamma)$

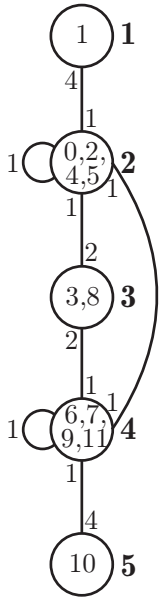

$\pi_{2}(\Gamma)$

FiguRE 1. Left: The walk-regular, but not distance-regular, graph $\Gamma$ given by Godsil [21]. Center and right: The quotient graphs $\pi_{1}(\Gamma)$ and $\pi_{2}(G)$ showing the vertices in each of the classes of the regular partitions. In boldface, there is the numbering of the vertices in $\Gamma, \pi_{1}(\Gamma)$, and $\pi_{2}(\Gamma)$.

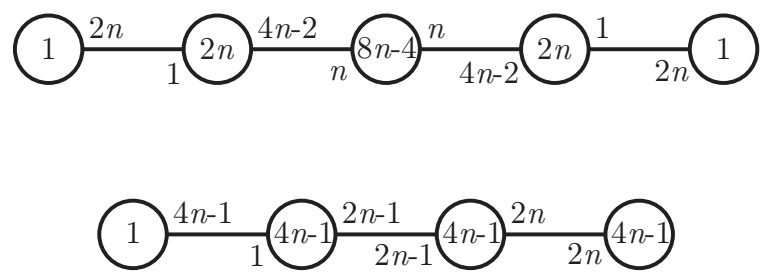

FIGURE 2. The quotient graphs of the Hadamard distance-biregular graphs.

4.3. Distance-biregular graphs. Distance-biregular graphs are defined in a similar way as distanceregular graphs. They are connected bipartite graphs in which each of the two classes of vertices has its own intersection array. It was proved by Godsil and Shawe-Taylor [23] that all vertices in the same bipartition class have the same intersection array. Delorme [12] gave the basic properties and some new examples of distance-biregular graphs.

Let us give an example.

EXAmple 4.3. A Hadamard matrix $\boldsymbol{H}$ (with entries \pm 1 and mutually orthogonal rows) with size $4 n$ gives a bipartite distance-biregular graph $H b(n)$ on $12 n-2$ vertices. To obtain the graph $H b(n)$ from the matrix $\boldsymbol{H}$, we apply the following steps: 
1. Given the Hadamard matrix

$$
\boldsymbol{H}(4 n)=\left(\begin{array}{c|ccc}
1 & 1 & \cdots & 1 \\
\hline 1 & & \\
\vdots & & \boldsymbol{D} & \\
1 & &
\end{array}\right)_{4 n \times 4 n}
$$

consider the square matrix $\boldsymbol{D}$ of dimension $4 n-1$.

2. Let $\boldsymbol{C}$ be the matrix $\boldsymbol{C}=\frac{1}{2}(\boldsymbol{D}+\boldsymbol{J})_{(4 n-1) \times(4 n-1)}$, where $\boldsymbol{J}$ is the all-1 matrix.

3. Consider the matrix

$$
\boldsymbol{B}=\left(\begin{array}{cc}
\boldsymbol{C} & \boldsymbol{J}-\boldsymbol{C} \\
\mathbf{1}^{\top} & \mathbf{0}^{\top}
\end{array}\right)_{4 n \times(8 n-2)},
$$

where $\mathbf{1}$ and $\mathbf{0}$ are the all- 1 and all-0 vectors with the corresponding dimensions.

4. Finally, the adjacency matrix of the Hadamard bipartite distance-biregular graph is

$$
\boldsymbol{A}=\left(\begin{array}{cc}
\boldsymbol{O} & \boldsymbol{B} \\
\boldsymbol{B}^{\top} & \boldsymbol{O}
\end{array}\right)_{(12 n-2) \times(12 n-2)} .
$$

For more information, see, for instance, Seberry Wallis [29, p. 426].

The stable sets $V_{1}$ and $V_{2}$ of $H b(n)$ have $4 n$ and $8 n-2$ vertices, respectively. For example, $H b(1)$ is the subdivided complete graph $K_{4}$. The quotient graphs corresponding to the regular distance-partitions with respect to vertices in $V_{1}$ and $V_{2}$ are shown in Figure 2. Thus, the respective quotient matrices are

$$
\boldsymbol{B}_{1}=\left(\begin{array}{ccccc}
0 & 2 n & 0 & 0 & 0 \\
1 & 0 & 4 n-2 & 0 & 0 \\
0 & n & 0 & n & 0 \\
0 & 0 & 4 n-2 & 0 & 1 \\
0 & 0 & 0 & 2 n & 0
\end{array}\right), \quad \boldsymbol{B}_{2}=\left(\begin{array}{cccc}
0 & 4 n-1 & 0 & 0 \\
1 & 0 & 2 n-1 & 0 \\
0 & 2 n-1 & 0 & 2 n \\
0 & 0 & 2 n & 0
\end{array}\right)
$$

with (simple) eigenvalues ev $\boldsymbol{B}_{1}=\left\{\sqrt{8 n^{2}-2 n}, \sqrt{2 n}, 0,-\sqrt{2 n},-\sqrt{8 n^{2}-2 n}\right\}$ and ev $\boldsymbol{B}_{2}=$ ev $\boldsymbol{B}_{1} \backslash\{0\}$. Then, according to Theorem 2.4, we can compute all the local (crossed) multiplicities of vertices in each stable set from the idempotents of $\boldsymbol{B}_{1}$ and $\boldsymbol{B}_{2}$. The results obtained are shown in Table 1 (for $u \in V_{1}$ ) and Table 2 (for $u \in V_{2}$ ), where the last row in both tables corresponds to the sums in (1.5) for $\ell=0$ (or, for the case $u=v$, to (1.3)). Moreover, from the columns of local multiplicities (dist $(u, v)=0$ ), we can find the (global) multiplicities by using (1.4), which in our case becomes

$$
m\left(\theta_{i}\right)=\sum_{u \in V_{1}} m_{u}\left(\theta_{i}\right)+\sum_{v \in V_{2}} m_{v}\left(\theta_{i}\right)=(8 n-2) \cdot m_{u}\left(\theta_{i}\right)+4 n \cdot m_{v}\left(\theta_{i}\right) .
$$

Then, the complete spectrum of the Hadamard distance-biregular graph turns out to be

$$
\operatorname{sp} H b(n)=\left\{{\sqrt{8 n^{2}-2 n}}^{1}, \sqrt{2 n}^{4 n-1}, 0^{4 n-2},-\sqrt{2 n}^{4 n-1},-{\sqrt{8 n^{2}-2 n}}^{1}\right\} .
$$

Acknowledgments. The authors sincerely acknowledge the detailed reports of one of the reviewers, with valuable comments and suggestions that allowed us to improve the paper. 
459 A General Method to Obtain the Spectrum and Local Spectra of a Graph from its Regular Partitions

\begin{tabular}{|c|ccccc|}
\hline $\operatorname{dist}(u, v), u \in V_{1}$ & 0 & 1 & 2 & 3 & 4 \\
\hline \hline$m_{u v}\left(\theta_{0}\right)$ & $\frac{1}{16 n-4}$ & $\frac{\sqrt{2}}{8 \sqrt{4 n^{2}-n}}$ & $\frac{1}{16 n-4}$ & $\frac{\sqrt{2}}{8 \sqrt{4 n^{2}-n}}$ & $\frac{1}{16 n-4}$ \\
$m_{u v}\left(\theta_{1}\right)$ & $\frac{1}{4}$ & $\frac{\sqrt{2}}{8 \sqrt{n}}$ & 0 & $-\frac{\sqrt{2}}{8 \sqrt{n}}$ & $-\frac{1}{4}$ \\
$m_{u v}\left(\theta_{2}\right)$ & $\frac{2 n-1}{4 n-1}$ & 0 & $-\frac{1}{8 n-2}$ & 0 & $\frac{2 n-1}{4 n-1}$ \\
$m_{u v}\left(\theta_{3}\right)$ & $\frac{1}{4}$ & $-\frac{\sqrt{2}}{8 \sqrt{n}}$ & 0 & $\frac{\sqrt{2}}{8 \sqrt{n}}$ & $-\frac{1}{4}$ \\
$m_{u v}\left(\theta_{4}\right)$ & $\frac{1}{16 n-4}$ & $-\frac{\sqrt{2}}{8 \sqrt{4 n^{2}-n}}$ & $\frac{1}{16 n-4}$ & $-\frac{\sqrt{2}}{8 \sqrt{4 n^{2}-n}}$ & $\frac{1}{16 n-4}$ \\
\hline$\sum_{i=0}^{4} m_{u v}\left(\theta_{i}\right)$ & 1 & 0 & 0 & 0 & 0 \\
\hline
\end{tabular}

TABLE 1

Local multiplicities, from a vertex $u \in V_{1}$, of the Hadamard distance-biregular graph $H b(n)$.

\begin{tabular}{|c|cccc|}
\hline $\operatorname{dist}(u, v), u \in V_{2}$ & 0 & 1 & 2 & 3 \\
\hline \hline$m_{u v}\left(\theta_{0}\right)$ & $\frac{1}{8 n}$ & $\frac{\sqrt{2}}{8 \sqrt{4 n^{2}-n}}$ & $\frac{1}{8 n}$ & $\frac{\sqrt{2}}{8 \sqrt{4 n^{2}-n}}$ \\
$m_{u v}\left(\theta_{1}\right)$ & $\frac{4 n-1}{8 n}$ & $\frac{\sqrt{2}}{8 \sqrt{n}}$ & $-\frac{1}{8 n}$ & $-\frac{\sqrt{2}}{8 \sqrt{n}}$ \\
$m_{u v}\left(\theta_{2}\right)$ & 0 & 0 & 0 & 0 \\
$m_{u v}\left(\theta_{3}\right)$ & $\frac{4 n-1}{8 n}$ & $-\frac{\sqrt{2}}{8 \sqrt{n}}$ & $-\frac{1}{8 n}$ & $\frac{\sqrt{2}}{8 \sqrt{n}}$ \\
$m_{u v}\left(\theta_{4}\right)$ & $\frac{1}{8 n}$ & $-\frac{\sqrt{2}}{8 \sqrt{4 n^{2}-n}}$ & $\frac{1}{8 n}$ & $-\frac{\sqrt{2}}{8 \sqrt{4 n^{2}-n}}$ \\
\hline$\sum_{i=0}^{3} m_{u v}\left(\theta_{i}\right)$ & 1 & 0 & 0 & 0 \\
\hline
\end{tabular}

TABLE 2

Local multiplicities, from a vertex $u \in V_{2}$, of the Hadamard distance-biregular graph $\operatorname{Hb}(n)$.

\section{REFERENCES}

[1] N. Biggs. Algebraic Graph Theory. Cambridge University Press, Cambridge, 1974; second edition, 1993.

[2] R.A. Beezer. Orbit polynomial graphs of prime order. Discrete Math., 67:139-147, 1987.

[3] A.E. Brouwer and W.H. Haemers. Spectra of Graphs. Universitext, Springer, New York, 2012.

[4] M. Cámara, J. Fàbrega, M.A. Fiol, and E. Garriga. Some families of orthogonal polynomials of a discrete variable and their applications to graphs and codes. Electron. J. Combin., 16:\#R83, 2009.

[5] D.M. Cvetković and M. Doob. Developments in the theory of graph spectra. Linear Multilinear Algebra, 18:153-181, 1985.

[6] D. Cvetković, M. Doob, and H. Sachs. Spectra of Graphs. Theory and Applications (third edition). Johann Ambrosius Barth, Heidelberg, 1995.

[7] D. Cvetković, P. Rowlinson, and S. Simić. An Introduction to the Theory of Graph Spectra. London Mathematical Society Student Texts, Vol. 75, Cambridge University Press, Cambridge, 2010.

[8] C. Dalfó. A survey on the missing Moore graph. Linear Algebra Appl., 569:1-14, 2019.

[9] C. Dalfó and M.A. Fiol. A note on the order of iterated line digraphs. J. Graph Theory, 85(2):395-399, 2017.

[10] C. Dalfó, M.A. Fiol, and E. Garriga. A differential approach for bounding the index of graphs under perturbations. Electron. J. Combin., 18:\#P172, 2011.

[11] E.R. van Dam and W.H. Haemers. Developments on spectral characterizations of graphs. Discrete Math., 309:576-586, 2009.

[12] C. Delorme. Distance biregular bipartite graphs. European J. Combin., 15:223-238, 1994. 
[13] P. Delsarte. An algebraic approach to the association schemes of coding theory. Philips Res. Rep. Suppl., $10,1973$.

[14] P. Delsarte and V.I. Levenshtein. Association schemes and coding theory. IEEE Trans. Inform. Theory, 44(6):2477-2504, 1998.

[15] M.A. Fiol. Algebraic characterizations of distance-regular graphs. Discrete Math., 246:111-129, 2002.

[16] M.A. Fiol. Quotient polynomial graphs. Linear Algebra Appl., 488:363-376, 2016.

[17] M.A. Fiol and E. Garriga. From local adjacency polynomials to locally pseudo-distance-regular graphs. J. Combin. Theory Ser. B, 71:162-183, 1997.

[18] M.A. Fiol and E. Garriga. On the algebraic theory of pseudo-distance-regularitiy around a set. Linear Algebra Appl., 298:115-141, 1999.

[19] M.A. Fiol, E. Garriga, and J.L.A. Yebra. Boundary graphs: The limit case of a spectral property. Discrete Math., 226:155-173, 2001.

[20] M.A. Fiol and E. Garriga. An algebraic characterization of completely regular codes in distance-regular graphs. SIAM J. Discrete Math., 15(1):1-13, 2002.

[21] C.D. Godsil. Algebraic Combinatorics. Chapman and Hall, New York, 1993.

[22] C.D. Godsil and B.D. McKay. Feasibility conditions for the existence of walk-regular graphs. Linear Algebra Appl., 30:51-61, 1980.

[23] C.D. Godsil and J. Shawe-Taylor. Distance-regularised graphs are distance-regular or distance-biregular. J. Combin. Theory Ser. B, 43(1):14-24, 1987.

[24] H. Kurihara. An excess theorem for spherical 2-designs. Des. Codes Cryptogr., 65(1-2):89-98, 2012.

[25] L. Lovász. On the Shannon capacity of a graph. IEEE Trans. Inform. Theory, 25(1):1-7, 1979.

[26] N. Macon and A. Spitzbart. Inverses of Vandermonde matrices. Amer. Math. Monthly, 65(2):95-100, 1958.

[27] A. Moradiamani, M.A. Fiol, M. Jalili, L. Stone, G. Chen, and X. Yu. Discovering vital nodes for Laplacian spectra of complex networks based on Laplacian spectra. Submitted, 2019.

[28] A. Schrijver. New code upper bounds from the Terwilliger algebra and semidefinite programming. IEEE Trans. Inform. Theory, 51(8)2859-2866, 2005.

[29] J. Seberry Wallis. Hadamard matrices. In: W.D. Wallis, A.P. Street, and J. Seberry Wallis, Combinatorics: Room Squares, Sum-Free Sets, Hadamard Matrices, Lect. Notes Math., Springer-Verlag, Heildelberg, 292:274-489, 1972. 